\title{
Optimized algorithm in solid thyroid nodule elastography
}

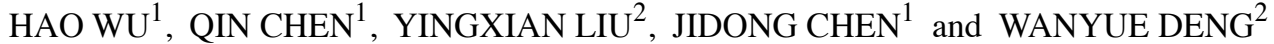 \\ ${ }^{1}$ Ultrasound Department, Sichuan Academy of Medical Sciences and Sichuan Provincial People's Hospital, \\ University of Electronic Science and Technology of China, Chengdu, Sichuan 610072; \\ ${ }^{2}$ Ultrasound Department, Emergency General Hospital, Beijing 100028, P.R. China
}

Received January 28, 2020; Accepted August 11, 2020

DOI: $10.3892 / \mathrm{ol} .2020 .12111$

\begin{abstract}
The present study aimed to evaluate the reproducibility and accuracy of the optimized algorithm of shear-wave elastography (SWE) in diagnosing solid thyroid nodules. Two hundred and sixty-three solid thyroid nodules in 248 patients who underwent conventional ultrasound and SWE, respectively, by two operators were scheduled for fine-needle aspiration or surgery. Elasticity indices of the mean, minimum and maximum of nodules (EI) and thyroid parenchyma (EInorm) were measured respectively in the same frame of elastographic images for three times by both operators. The intraobserver and interobserver reproducibility of the optimized algorithm were assessed by intraclass correlation coefficients (ICC). Diagnostic performance of the optimized algorithm was compared with that of conventional SWE measurements by receiver-operating characteristic (ROC) curves. Among a total of 243 nodules included, 121 were benign nodules and 122 were papillary thyroid carcinoma (PTC). Intraobserver reliability for EId and EIr was nearly perfect (ICC $>0.80)$. Interobserver agreement for MEANd, MAXd, MEANr and MAXr was nearly perfect (ICC $>0.80$ ). MAXd had the largest areas under the ROC curve which was 0.82 . Compared with conventional SWE, the
\end{abstract}

Correspondence to: Dr Qin Chen, Ultrasound Department, Sichuan Academy of Medical Sciences and Sichuan Provincial People's Hospital, University of Electronic Science and Technology of China, 32 West Section 2, First Ring Road, Chengdu, Sichuan 610072, P.R. China

E-mail:pq9t60@163.com

Abbreviations: USE, ultrasound elastography; SWE, shear wave elastography; FNA, fine-needle aspiration; ICC, intraclass correlation coefficients; PTC, papillary thyroid carcinoma; BHN, benign hyperplastic nodule; ROC curve, receiver-operating characteristic curve; AUC, areas under the ROC curve; $\mathrm{kPa}$, kilopascal; EI \& EInorm, elasticity indices (of the nodule and thyroid parenchyma); EId \& EIr=EI-EInorm \& EI/EInorm difference value and ratio value of elasticity indices between nodule and surrounding thyroid parenchyma; EIavg \& EInorm_avg, average value of three measurements for EI \& EInorm; EId_avg \& EIr_avg, average value of three measurements for EId \& EIr

Key words: shear-wave elastography, thyroid nodule, optimized algorithm, reproducibility, diagnostic accuracy optimized algorithm of SWE shows better reproducibility and performance in diagnosing solid thyroid nodules.

\section{Introduction}

In recent years we have witnessed an increasing trend in the incidence of thyroid nodules (1). However, only about 5 to $15 \%$ of the nodules are malignant. The optimal imaging method is a common diagnostic tool in thyroid diseases. The accuracy of optimal imaging method in the diagnosis of thyroid diseases needs further improvement. These improvements include improving the sonographic features of both benign and malignant thyroid nodules $(2,3)$.

Ultrasound elastography (USE) is a novel technology to qualitatively or quantitatively, measure the stiffness of the tissue that can offer valuable diagnostic information. This method is considered a promising tool in differential diagnostic of benign and malignant nodules. USE can be generally divided into two categories: Strain elastography and shear wave elastography (SWE). The former which emerged earlier needs an external force to generate tissue strain and has been proven to be helpful in the diagnosis of lesions in many organs such as the liver, breast and thyroid (4-7). This technology largely depends on the operator's experience which includes an interobserver reproducibility effect. SWE, which we used in this study, is a real-time elastography to measure the stiffness of the tissue qualitatively and quantitatively $(8,9)$. This is because the external force applied in SWE is generated by the sound pulse of the SWE system, and thus it has good reliability and repeatability.

Recently, SWE is the focus of elastography research which has demonstrated higher performances in diagnosing breast and thyroid lesions compared with conventional US and other existing elastography used in prior studies. However, the clinical application of SWE in thyroid lesions remains controversial (10-13). On the other hand, a recent study (14) demonstrated that the lesion elasticity (stiffness) is effected by the compression put on the probe although which is not necessary. That was similarly found in our study. We found that the discrepancy in the optimized algorithm, difference value and ratio value between nodules and thyroid parenchyma acquired in the same frame of elastographic images, was much smaller than that of conventional elasticity indices (EIs).

The aim of the present study was to evaluate the reproducibility and accuracy of the optimized algorithm of SWE in differentiating benign and malignant solid thyroid nodules. 


\section{Patients and methods}

Patients. From April 2016 to April 2017, 263 solid thyroid nodules in 248 patients (76 males and 152 females) were collected at the Sichuan Academy of Medical Sciences and Sichuan Provincial People's Hospital, University of Electronic Science and Technology of China. These solid thyroid nodules underwent conventional US and SWE by an operator with 8 years of experience in thyroid US examination and one year experience in SWE. The second operator with less experience in both methods conducted SWE immediately after the former one and was blind to previous results. Then all the patients were scheduled for ultrasound-guided fine-needle aspiration (FNA). Nodules with malignant or suspicious cytopathology received surgery and part of the benign nodules $(n=54)$ received surgery as well. Twenty of the 263 nodules were excluded due to unqualified cytopathologic results. The study was approved by the Ethics Committee of Sichuan Academy of Medical Sciences and Sichuan Provincial People's Hospital, China. Patients who participated in this research, signed the informed consent and had complete clinical data.

ConventionalUSexamination. ConventionalUS wasperformed using a 4-15 MHz linear transducer (SuperLinear ${ }^{\mathrm{TM}}$ SL 15-4) on the AIXPLORER system (Aixplorer, SuperSonic Imagine, Aix-en-Provence). This provided real-time assessment of tissues stiffness with the SWE color map qualitatively and elasticity modulus (Young's modulus) quantitatively. Patients were positioned in a supine position with their necks bend back slightly over a pillow. The nodule size, depth (distance from the skin), location (isthmus or not) and calcification (yes or no) were observed and recorded by the first operator. Only the solid nodules with the size $<30 \mathrm{~mm}$ and depth $<25 \mathrm{~mm}$ and without macrocalcification were included in this study.

SWE examination. After receiving conventional US examination, patients underwent SWE in the same position by the same operator using the same transducer. Subsequently, SWE mode was activated and the region of interest (ROI) was applied on the longitudinal view of the nodule with maximum diameter (Fig. 1). ROI was the SWE color map box in which the blue area to red area represented lowest stiffness region to highest stiffness region with Young's modulus ranging from 0-140 kPa and the target nodule and surrounding thyroid parenchyma was included. The probe was held for about $5 \mathrm{sec}$ on the patient's neck without applying any pressure with adequate amount of transducer gel on the skin above the nodule in order to stabilize the SWE images. Subsequently, the best frame with a reasonable color map covering the target region without filling defect of the saved images was selected to measure the mean, minimum and maximum (showed as Emean, Emin and Emax on the AIXPLORER system) of elasticity indices of the nodule (recorded as EIs, including MEAN, MIN and MAX) and thyroid parenchyma (recorded as EInorm, including MEANnorm, MINnorm and MAXnorm) by two circular sampling volume $\left(\mathrm{Q}-\mathrm{Box}^{\mathrm{TM}}\right)$ respectively. The first $\mathrm{Q}-\mathrm{Box}^{\mathrm{TM}}$ was placed in the target nodule with a size equal to the size of the whole nodule while the tissues outside the nodule were not included (eg. strap muscles, trachea). The second Q-Box ${ }^{\mathrm{TM}}$ with the same size of $2 \mathrm{~mm}$ was placed in the surrounding
Table I. Characteristics of the 243 thyroid solid nodules.

\begin{tabular}{lcc}
\hline Characteristics & Benign (n) & Malignant (n) \\
\hline Total no. of nodules & 121 & 122 \\
Size (mm) & & \\
$\leq 10$ & 87 & 63 \\
$10-20$ & 28 & 53 \\
$>20$ & 6 & 6 \\
Depth (mm) & & \\
$\leq 10$ & 64 & 75 \\
$10-20$ & 57 & 46 \\
$>20$ & 0 & 1 \\
Location & & \\
Isthmus & 19 & 33 \\
Not isthmus & 102 & 89 \\
Calcification & & \\
Yes & 53 & 74 \\
No & 88 & 48 \\
\hline
\end{tabular}

thyroid parenchyma. The EI and EInorm were measured and recorded for three times and before each measurement, the transducer was removed and reapplied. The average value of three measurements for EI and EInorm was recorded as EIavg (including MEANavg, MINavg and MAXavg) and EInorm_avg (including MEANnorm_avg, MINnorm_avg and MAXnorm_avg), respectively. The second operator repeated SWE examination immediately after the first operator and was blind to previous results.

Optimized algorithm. The optimized algorithm was the difference in the value of elasticity modulus between the nodule and surrounding thyroid parenchyma in the same frame of SWE images (EId=EI-EInorm, including MEANd, MINd and MAXd and EId_avg=EIavg-EInorm_avg, including MEANd_avg, MINd_avg and MAXd_avg) and the ratio value of elasticity modulus between the nodule and surrounding thyroid parenchyma measured in the same frame of SWE images (EIr=EI/EInorm, including MEANr, MINr and MAXr and EIr_avg=EIavg/EInorm_avg, including MEANr_avg, MINr_avg and MAXr_avg).

Statistical analysis. All the data were analyzed using SPSS statistical software (IBM Corp.) version 19.0. The intraclass correlation coefficients (ICCs) were calculated to measure the reliability of using a one-way analysis of variance (ANOVA). The ICCs of EId and EIr calculated by the first observer were measured for intraobserver reliability. The EId_avg and EIr_avg calculated by both observers were measured for interobserver reliability. An ICC of 0-0.49 indicates poor reliability; 0.50-0.74 indicates moderate reliability; 0.75-1.00 indicates excellent reliability based on the criteria (15). Receiver-operating characteristic (ROC) analyses were performed to evaluate the diagnostic performances of conventional SWE parameters and optimized algorithm parameters and the areas under the ROC curves (AUCs) were 
Table II. Intraobserver reliability of the optimized algorithm measurements for 243 solid thyroid nodules for the first operator.

\begin{tabular}{|c|c|c|c|c|c|c|}
\hline \multirow[b]{2}{*}{ Parameters } & \multicolumn{2}{|c|}{ Benign } & \multicolumn{2}{|c|}{ Malignant } & \multicolumn{2}{|c|}{ Total } \\
\hline & ICC & $95 \% \mathrm{CI}$ & ICC & $95 \%$ CI & ICC & $95 \%$ CI \\
\hline MEANd $^{\mathrm{a}}$ & 0.91 & $0.88-0.94$ & 0.91 & $0.88-0.94$ & 0.93 & $0.92-0.95$ \\
\hline $\mathrm{MINd}^{\mathrm{b}}$ & 0.77 & $0.69-0.84$ & 0.88 & $0.84-0.91$ & 0.86 & 0.83-0.89 \\
\hline $\mathrm{MAXd}^{\mathrm{c}}$ & 0.94 & $0.92-0.96$ & 0.89 & $0.85-0.92$ & 0.92 & $0.91-0.94$ \\
\hline $\mathrm{MEANr}^{\mathrm{d}}$ & 0.87 & $0.83-0.91$ & 0.89 & $0.85-0.92$ & 0.91 & $0.89-0.93$ \\
\hline $\mathrm{MINr}^{\mathrm{e}}$ & 0.81 & $0.74-0.86$ & 0.91 & $0.88-0.94$ & 0.90 & $0.87-0.92$ \\
\hline MAXr ${ }^{\mathrm{f}}$ & 0.90 & $0.87-0.93$ & 0.86 & $0.82-0.90$ & 0.91 & $0.88-0.92$ \\
\hline
\end{tabular}

Parameters were measured by the first operator. ${ }^{2}$ MEANd is the difference value of mean elasticity modulus between the nodule and surrounding thyroid parenchyma. ${ }^{b} \mathrm{MINd}$ is the difference value of min elasticity modulus between the nodule and surrounding thyroid parenchyma. ${ }^{\mathrm{c}} \mathrm{MAXd}$ is the difference value of max elasticity modulus between the nodule and surrounding thyroid parenchyma. ${ }^{\mathrm{d}} \mathrm{MEANr}$ is the ratio value of mean elasticity modulus between the nodule and surrounding thyroid parenchyma. ${ }^{\mathrm{e}} \mathrm{MINr}$ is the ratio value of min elasticity modulus between the nodule and surrounding thyroid parenchyma. ${ }^{\mathrm{f}} \mathrm{MAXr}$ is the ratio value of max elasticity modulus between the nodule and surrounding thyroid parenchyma. ICC, intraclass correlation coefficients; CI, confidence interval.
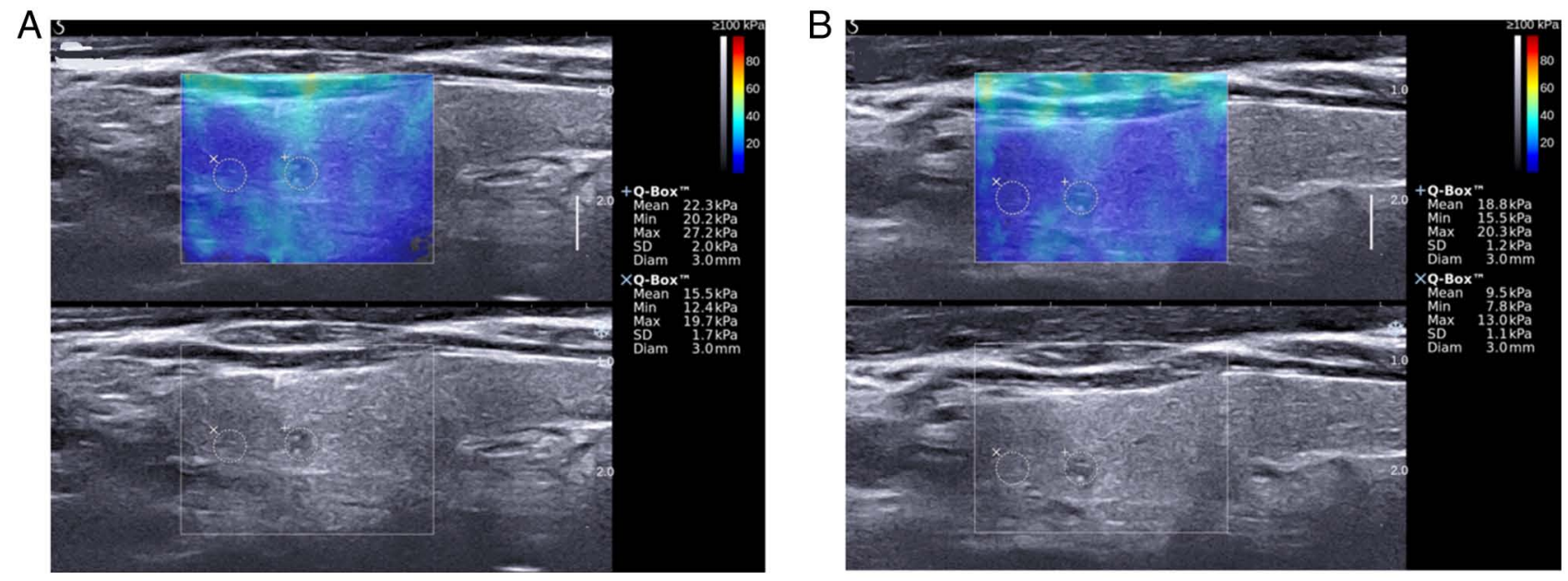

Figure 1. The longitudinal view of Shear-wave elastography (SWE) images of nodular goiter acquired (A) with or (B) without precompression in the same lesion. Each of the elasticity indices (EIs) measured with precompression was apparently increased. But MAXd (difference value of Emax between nodules and thyroid parenchyma) acquired with precompression (a, MAXd=7.5 kPa) was not so obviously different from that acquired without precompression (b, MAXd=7.3 kPa).

calculated to determine the best diagnostic cut-off value for each parameter. In addition, the optimal SWE parameter and its corresponding sensitivity, specificity, accuracy, positive predictive value (PPV) and negative predictive value (NPV) were calculated.

\section{Results}

Two hundred and forty-eight patients (age range, 5-85 years; mean age: 43.18 years) with 243 solid nodules were included in this study (Table I). The mean size of the nodules was $9.83 \pm 5.15 \mathrm{~mm}$ (range, 2.90-29.50 $\mathrm{mm}$ ). The benign nodules $(n=121)$ including nodular goiter $(n=44)$, adenoma $(n=4)$, focal thyroiditis $(n=35)$, and other benign hyperplastic nodule $(n=38)$ were confirmed by qualified FNA cytology tests or surgery. All of the malignant nodules $(n=122)$ were confirmed as papillary thyroid carcinoma (PTC) by surgery.
Intraobserver reproducibility of the optimized algorithm for 243 solid thyroid nodules. The intraobserver reliability of the optimized algorithm for benign nodules was nearly perfect for MEANd, MAXd, MEANr, MINr and MAXr with ICC $>0.80$ and MINd was substantial with ICC $=0.77$ (Table II). For malignant nodules, the reliability was nearly perfect for all parameters with ICC $>0.80$. As for all 243 nodules, the reliability was nearly perfect for all parameters with ICC $>0.80$.

Interobserver reproducibility of the optimized algorithm for 243 solid thyroid nodules. In the benign nodules, the interobserver agreement for MEANd_avg, MAXd_avg, MEANr_avg and MAXr_avg was nearly perfect with ICC $>0.80$ and was substantial for MINd_avg and MINr_avg with ICC $=0.72$ and 0.71 , respectively (Table III). In the malignant nodules, it was nearly perfect for all parameters with ICC $>0.80$. As for all 243 nodules, the interobserver agreement was nearly 
Table III. Interobserver reliability of the optimized algorithm measurements for 243 solid thyroid nodules.

\begin{tabular}{|c|c|c|c|c|c|c|}
\hline \multirow[b]{2}{*}{ Parameters } & \multicolumn{2}{|c|}{ Benign } & \multicolumn{2}{|c|}{ Malignant } & \multicolumn{2}{|c|}{ Total } \\
\hline & ICC & $95 \% \mathrm{CI}$ & $\mathrm{ICC}$ & $95 \% \mathrm{CI}$ & $\mathrm{ICC}$ & $95 \% \mathrm{CI}$ \\
\hline MEANd_avg ${ }^{\mathrm{a}}$ & 0.86 & $0.81-0.90$ & 0.90 & $0.86-0.93$ & 0.91 & $0.89-0.93$ \\
\hline MINd_avg ${ }^{b}$ & 0.72 & $0.62-0.80$ & 0.79 & $0.71-0.84$ & 0.78 & $0.72-0.82$ \\
\hline MAXd_avg ${ }^{c}$ & 0.89 & $0.85-0.92$ & 0.89 & $0.84-0.92$ & 0.91 & $0.89-0.93$ \\
\hline MEANr_avg ${ }^{\mathrm{d}}$ & 0.80 & $0.72-0.86$ & 0.86 & $0.80-0.90$ & 0.87 & $0.83-0.90$ \\
\hline MINr_avg ${ }^{\mathrm{e}}$ & 0.71 & $0.61-0.79$ & 0.80 & $0.73-0.86$ & 0.79 & $0.74-0.83$ \\
\hline MAXr_avg ${ }^{f}$ & 0.83 & $0.77-0.88$ & 0.79 & $0.71-0.85$ & 0.84 & $0.80-0.87$ \\
\hline
\end{tabular}

Parameters were measured by the first operator. ${ }^{a} \mathrm{MEANd} \_$avg is the average difference value of three measurements for mean elasticity modulus between the nodule and surrounding thyroid parenchyma. ${ }^{b} \mathrm{MINd}$ avg is the average difference value of three measurements for min elasticity modulus between the nodule and surrounding thyroid parenchyma. ${ }^{\mathrm{M}} \mathrm{MAXd}$ avg is the average difference value of three measurements for max elasticity modulus between the nodule and surrounding thyroid parenchyma. ${ }^{\mathrm{d}} \mathrm{MEANr}$ avg is the average ratio value of three measurements for mean elasticity modulus between the nodule and surrounding thyroid parenchyma. ${ }^{\mathrm{e}} \mathrm{MINr}$ avg is the average ratio value of three measurements for min elasticity modulus between the nodule and surrounding thyroid parenchyma. ${ }^{\mathrm{f}} \mathrm{MAXr} \_$avg is the average ratio value of three measurements for max elasticity modulus between nodule and surrounding thyroid parenchyma. ICC, intraclass correlation coefficients; CI, confidence interval.

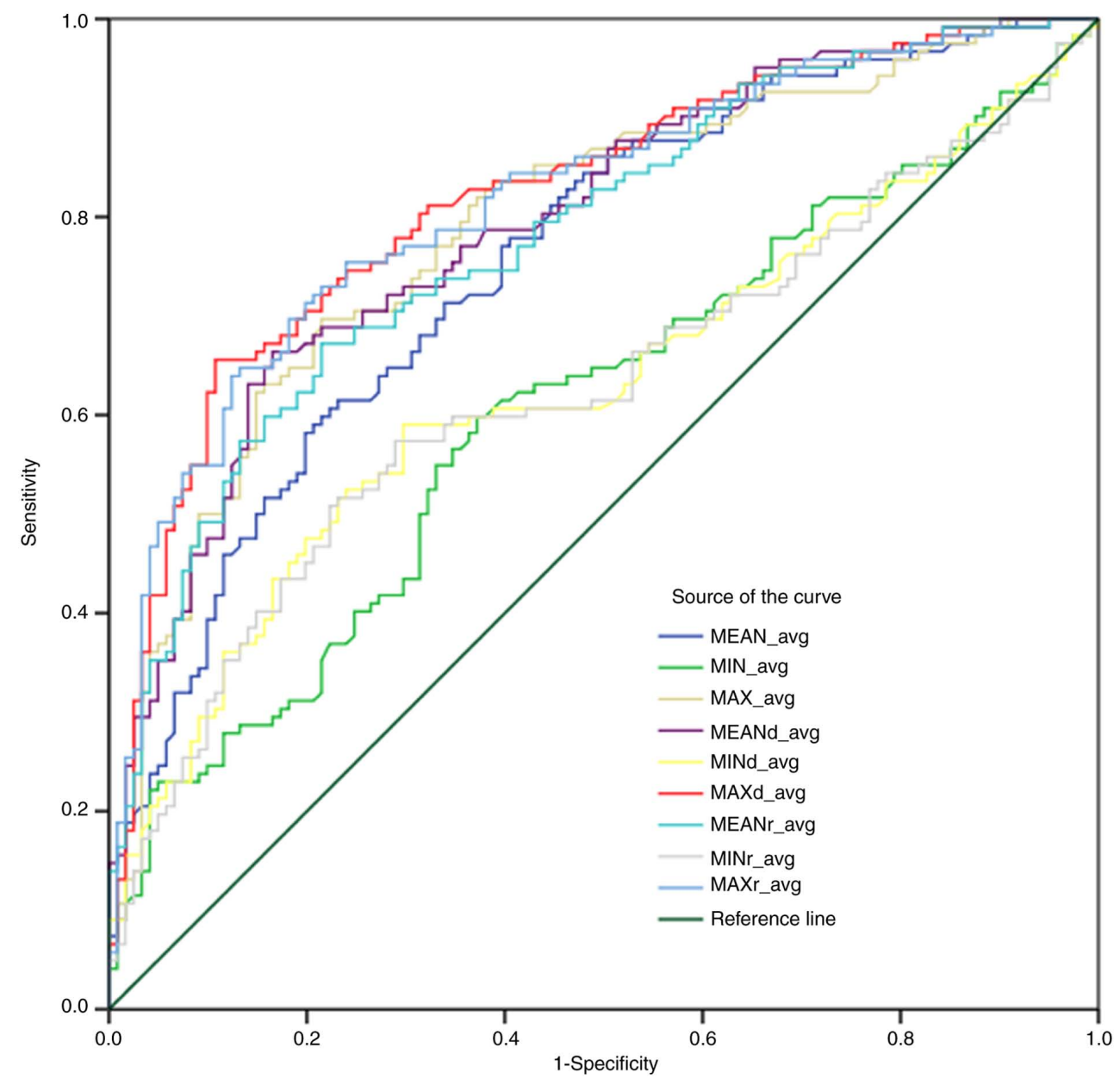

Figure 2. Receiver operating characteristic (ROC) curves (area under the ROC curve) for conventional Shear-wave elastography (SWE) parameters and optimized algorithm parameters.

perfect for MEANd_avg, MAXd_avg, MEANr_avg and MAXr_avg with ICC $>0.80$ and was substantial for MINd_avg and MINr_avg with ICC $=0.78$ and 0.79 , respectively.
Accuracy of the optimized algorithm. The ROC curves for parameters in the optimized algorithm is shown in Fig. 2. This suggested that the MAXd_avg had more sensitivity and 
Table IV. Comparison of the diagnostic performances of conventional SWE and optimized algorithm parameters.

\begin{tabular}{lccccccc}
\hline Parameters & Cut-off value & Sensitivity (\%) & Specificity (\%) & Accuracy (\%) & PPV (\%) & NPV (\%) & AUC (95\% CI) \\
\hline MEAN_avg $^{\mathrm{a}}$ & $38.85 \mathrm{kPa}$ & 58.20 & 80.17 & 69.14 & 74.74 & 65.54 & $0.76(0.70-0.82)$ \\
MIN_avg $^{\mathrm{b}}$ & $18.11 \mathrm{kPa}$ & 59.84 & 62.81 & 61.32 & 61.86 & 60.80 & $0.61(0.54-0.68)$ \\
MAX_avg $^{\mathrm{c}}$ & $61.55 \mathrm{kPa}$ & 69.67 & 78.51 & 74.07 & 76.58 & 71.97 & $0.79(0.74-0.85)$ \\
MEANd_avg $^{\mathrm{d}}$ & $14.30 \mathrm{kPa}$ & 66.39 & 83.47 & 74.90 & 81.20 & 71.13 & $0.80(0.74-0.85)$ \\
MINd_avg $^{\mathrm{c}}$ & $1.93 \mathrm{kPa}$ & 59.02 & 70.25 & 64.61 & 66.67 & 62.96 & $0.63(0.56-0.70)$ \\
MAXd_avg & $32.95 \mathrm{kPa}$ & 65.57 & 89.26 & 77.37 & 86.02 & 72.0 & $0.82(0.77-0.87)$ \\
MEANr_avg & 1.61 & 67.21 & 78.51 & 72.84 & 75.93 & 70.37 & $0.78(0.73-0.84)$ \\
MINr_avg & 1.22 & 50.82 & 77.69 & 64.20 & 69.66 & 61.04 & $0.63(0.56-0.70)$ \\
MAXr_avg & 2.15 & 63.93 & 87.60 & 75.72 & 83.87 & 70.67 & $0.82(0.76-0.87)$ \\
\hline
\end{tabular}

${ }^{\mathrm{a}} \mathrm{MEAN} \_$avg is the average value of three measurements for mean elasticity modulus of the nodule. ${ }^{\mathrm{b}} \mathrm{MIN} \_$avg is the average value of three measurements for min elasticity modulus of the nodule. ${ }^{\mathrm{C}} \mathrm{MAX}$ _avg is the average value of three measurements for max elasticity modulus of the nodule. ${ }^{\mathrm{d}} \mathrm{MEANd} \_$avg is the average difference value of three measurements for mean elasticity modulus between the nodule and surrounding thyroid parenchyma. ${ }^{\mathrm{e}} \mathrm{MINd} \_$avg is the average difference value of three measurements for min elasticity modulus between the nodule and surrounding thyroid parenchyma. ${ }^{\mathrm{f}} \mathrm{MAXd}$ _avg is the average difference value of three measurements for max elasticity modulus between the nodule and surrounding thyroid parenchyma. ${ }^{9} \mathrm{MEANr} \_$avg is the average ratio value of three measurements for mean elasticity modulus between the nodule and surrounding thyroid parenchyma. ${ }^{\mathrm{h}} \mathrm{MINr}$ _avg is the average ratio value of three measurements for min elasticity modulus between the nodule and surrounding thyroid parenchyma. ${ }^{i} \mathrm{MAXr} \_a v g$ is the average ratio value of three measurements for max elasticity modulus between the nodule and surrounding thyroid parenchyma. SWE, shear wave elastography; PPV, positive predictive value; NPV, negative predictive value; AUC, area under the ROC curves; CI, confidence interval.

specificity compared with others in predicting malignant nodules with the cut-off value of $32.95 \mathrm{kPa}$ and the sensitivity, specificity, accuracy, PPV and NPV were 65.57, 89.26, 77.37, 86.02 and 72.0, respectively (Table IV).

\section{Discussion}

Previous studies have demonstrated that the size, location and presence of calcification of thyroid nodules have a certain impact on their elasticity modulus (16). Another research study also indicated that partially cystic nodules had higher elasticity modules than solid ones in both shear wave elastography (SWE) and strain elastography although the cause remains unknown $(16,17)$. In addition, it was reported by Cengic et al (18) that the success rate of fine-needle aspiration (FNA) was lower and total biopsy time of FNA was longer in predominantly cystic nodules than that in predominantly solid ones. Furthermore, Gu et al (19) and Chan et al (20) reported that the risk of being malignant in solid or predominantly solid thyroid nodules was higher than that in the cystic or predominantly cystic nodules. Thus, the cases studied in the present report were all solid thyroid nodules and the main focus was the difficulty in differentiating between benign and malignant thyroid nodules.

Bhatia et al (17) and Szczepanek-Parulska et al (16) reported that the size of a nodule has a positive correlation with its elasticity modulus. In addition, researchers analyzed 382 nodules with size $\geq 4 \mathrm{~cm}$ and found that the incidence of a clinically significant thyroid carcinoma nodule was $22 \%$. Using these findings, they recommended that all nodules with a size $\geq 4 \mathrm{~cm}$ should be considered for either thyroid lobectomy or total thyroidectomy (21). Thus, an overlarge nodule appears to be an independent risk factor of thyroid carcinoma which is of little clinical significance for SWE examination and should be treated with more aggressive treatments. All nodules studied in our study were smaller than $30 \mathrm{~mm}$.

Prior reports on SWE for breast, liver and other organs disclosed that when the lesion is too deep, the accuracy of measurement decreases and the false-positive rate of elastography increases (22-25). Therefore, the depth of nodule was less than $25 \mathrm{~mm}$.

Almost every report published on this subject was unanimous on the verdict that calcification has a significant effect on elasticity. The elasticity modulus of a nodule can increase remarkably if both microcalcification and macrocalcification are present. Consequently, some researchers (16) considered that thyroid nodules with macrocalcification would not be appropriate for SWE examination (26). Thus, in our study the nodules with macrocalcification (size $>2 \mathrm{~mm}$ ) were excluded.

The conventional SWE parameters presents a great reliability in measurements of thyroid nodules and the optimized algorithm used in our study showed a similar performance. Intraclass correlation coefficients of EId and EIr of the first operator were almost perfect for all benign and malignant nodules (ICC $>0.75$ for each). ICCs between two operators for MEANd_avg, MEANr_avg, MAXd_avg and MAXr_avg were almost perfect (ICC $>0.8$ for each). This can be explained by the following facts: First, with the first sampling volume (the first Q-Box ${ }^{\mathrm{TM}}$ ) covering the entire target nodule in optimized algorithm, there could be no error on the choices of maximum elasticity region by different operators. Veyrieres et al (11) examined 297 thyroid nodules (35 of which were malignant nodules) using shear-wave elastography on the AIXPLORER system and found that the sensitivity of diagnosing malignant nodules was $80 \%$ and the specificity was $90.5 \%$ with the optimal cut-off value $(65 \mathrm{kPa})$ 
on the most stiffness region of the nodule. Kim et al (13) analyzed 99 nodules with the same cut-off value $(65 \mathrm{kPa})$ using the same method for sampling, but its sensitivity and specificity of diagnosis was much lower (76.1 and 64.1\%). Although Bhatia et al (27) and Liu et al (28) used the same sampling method $\left(2 \mathrm{~mm}\right.$ size of $\left.\mathrm{Q}-\mathrm{Box}^{\mathrm{TM}}\right)$ with a similar cut-off value (42.1 and $39.3 \mathrm{kPa})$ to predict malignancy, they discovered that their diagnostic performances were not quite consistent. This was due to the different proportions of pathologic results and their sampling volume (Q-Box $\left.{ }^{\mathrm{TM}}\right)$ that only covered the hardest region of the nodule. Secondly, in the present report, we report that each time we operated SWE, the compression on the transducer would significantly affect the measurement results (Fig. 1). Lam et al (14) pointed out that when the precompression increased by $22-30 \%$, the elasticity modulus of thyroid parenchyma, benign hyperplastic nodule (BHN) and papillary thyroid carcinoma (PTC) would increase simultaneously but differently $(10.8,24.6$ and $75.4 \mathrm{kPa}$ ). In addition, no matter how much precompression was exerted, there was a significant difference between the difference value of PTC vs. parenchyma and BNH vs. parenchyma, which was consistent with our results. We concluded that the optimized algorithm used in our study was able to avoid the influence of compression and had a high rate of reproducibility. Moreover, the MAXd variables in the optimization algorithm had a specificity of $89.26 \%$, which could exclude nodules that did not need fine-needle aspiration (FNA), thus avoiding invasive examination and waste of medical resources.

Shear-wave elastography (SWE) has been proven to be useful in differential diagnosis of thyroid nodules. In most previous studies, the diagnostic performance of the mean elasticity of the nodule (Emean) was slightly higher than that of the minimum elasticity (Emin) and maximum elasticity (Emax). Duan et al (29) reported that AUC of Emean (0.789) was greater than that of Emin (0.703) and Emax (0.701). However, the results of our study demonstrated that neither the AUC value of Emean of the nodule (MEAN_avg) nor the AUC value of MEANd_avg and MEANr_avg $(0.76,0.80$ and 0.78) was higher than that of MAX_avg, MAXd_avg and MAXr_avg (0.79, 0.82 and 0.82) (Table IV), which was different from previous studies. This difference may be due to the following reasons. First, in previous studies, the sampling volume, the size of which was fixed at $2 \mathrm{~mm}$, was placed in the most stiffest part of the target nodule and the mean elasticity modulus was close to the highest modulus of the entire nodule which was similar as the max elasticity modulus of the entire nodule measured by the method of our study. The difference in the MEAN or MEANavg in our study was influenced by the homogeneity of the nodule. Secondly, the diagnostic performance was also affected by the composition of the cases, such as pathology results, size, depth and other factors which would affect specificity, accuracy and PPV of MAXd_avg (cut-off value $=32.95 \mathrm{kPa}$ ) in the optimized algorithm was higher than either the conventional SWE indices or other indices in the optimized algorithm.

We acknowledge that the present study had several limitations. First, the placement of sampling volume for thyroid parenchyma was certainly influenced by the subjective factors. Secondly, the influence of chronic thyroiditis on elasticity (30-32) is still considered a controversial issue. However, there were no separate analyses on cases with subacute thyroiditis or Hashimoto thyroiditis which were included in this study. Thirdly, previous research (10) has confirmed that the elasticity modulus varies from different pathologic subtypes. However, all the malignant nodules in this study were PTC. Due to the insufficient data on specific pathological subtypes, they were not compared with variables in the optimization algorithm, which deserves further attention. In addition, it can be seen from previous studies that the size, depth and location of nodules are the significant influencing factors that have not been grouped and studied in our research.

In conclusion, the optimized algorithm of SWE elastography showed good reproducibility and better performance in diagnosing solid thyroid nodules than conventional SWE and the best elasticity indices in optimized algorithm was MAXd, the difference value of elasticity modulus between the nodule and surrounding thyroid parenchyma. However, the size, depth, location and calcification of the nodule had an important impact on SWE performance. Therefore, the conditions in which the optimized algorithm will work better requires further study.

\section{Acknowledgements}

Not applicable.

\section{Funding}

No funding was received.

\section{Availability of data and materials}

The datasets used and/or analyzed during the current study are available from the corresponding author on reasonable request.

\section{Authors' contributions}

HW designed the study and drafted the manuscript. YL and WD were responsible for the collection and analysis of the experimental data. QC and JC performed the conventional US examinations and revised the manuscript critically for important intellectual content. All authors read and approved the final manuscript.

\section{Ethics approval and consent to participate}

The study was approved by the Ethics Committee of Sichuan Academy of Medical Sciences and Sichuan Provincial People's Hospital, China. Patients who participated in this research, signed the informed consent and had complete clinical data.

\section{Patient consent for publication}

Not applicable.

\section{Competing interests}

The authors declare that they have no competing interests. 


\section{References}

1. Stanicić J, Prpić M, Jukić T, Borić M and Kusić Z: Thyroid nodularity-true epidemic or improved diagnostics. Acta Clin Croat 48: 413-418, 2009.

2. American Thyroid Association (ATA) Guidelines Taskforce on Thyroid Nodules and Differentiated Thyroid Cancer, Cooper DS, Doherty GM, Haugen BR, Kloos RT, Lee SL, Mandel SJ, Mazzaferri EL, McIver B, Pacini F, et al: Revised American Thyroid Association management guidelines for patients with thyroid nodules and differentiated thyroid cancer. Thyroid 19 $1167-1214,2009$

3. Guth S, Theune U, Aberle J, Galach A and Bamberger CM: Very high prevalence of thyroid nodules detected by high frequency (13 MHz) ultrasound examination. Eur J Clin Invest 39: 699-706, 2009.

4. Baker JA, Kornguth PJ, Soo MS, Walsh R and Mengoni P: Sonography of solid breast lesions: Observer variability of lesion description and assessment. AJR Am J Roentgenol 172 $1621-1625,1999$.

5. Lyshchik A, Higashi T, Asato R, Tanaka S, Ito J, Mai JJ, Pellot-Barakat C, Insana MF, Brill AB, Saga T, et al: Thyroid gland tumor diagnosis at US elastography. Radiology 237 202-211, 2005.

6. Itoh A, Ueno E, Tohno E, Kamma H, Takahashi H, Shiina T, Yamakawa $\mathrm{M}$ and Matsumura T: Breast disease: Clinical application of US elastography for diagnosis. Radiology 239: 341-350, 2006.

7. Chang JM, Moon WK, Cho $\mathrm{N}$ and Kim SJ: Breast mass evaluation: Factors influencing the quality of US elastography. Radiology 259: 59-64, 2011.

8. Bercoff J, Tanter M and Fink M: Supersonic shear imaging: A new technique for soft tissue elasticity mapping. IEEE Trans Ultrason Ferroelectr Freq Control 51: 396-409, 2004

9. Tanter M, Bercoff J, Athanasiou A, Deffieux T, Gennisson JL, Montaldo G, Muller M, Tardivon A and Fink M: Quantitative assessment of breast lesion viscoelasticity: Initial clinical results using supersonic shear imaging. Ultrasound Med Biol 34 1373-1386, 2008.

10. Sebag F, Vaillant-Lombard J, Berbis J, Griset V, Henry JF, Petit P and Oliver C: Shear wave elastography: A new ultrasound imaging mode for the differential diagnosis of benign and malignant thyroid nodules. J Clin Endocrinol Metab 95: 5281-5288, 2010.

11. Veyrieres JB, Albarel F, Lombard JV, Berbis J, Sebag F, Oliver C and Petit P: A threshold value in shear wave elastography to rule out malignant thyroid nodules: A reality? Eur J Radiol 81: 3965-3972, 2012.

12. Lin P, Chen M, Liu B, Wang S and Li X: Diagnostic performance of shear wave elastography in the identification of malignant thyroid nodules: A meta-analysis. Eur Radiol 24: 2729-2738, 2014

13. Kim H, Kim JA, Son EJ and Youk JH: Quantitative assessment of shear-wave ultrasound elastography in thyroid nodules: Diagnostic performance for predicting malignancy. Eur Radiol 23: 2532-2537, 2013.

14. Lam AC, Pang SW, Ahuja AT and Bhatia KS: The influence of precompression on elasticity of thyroid nodules estimated by ultrasound shear wave elastography. Eur Radiol 26: 2845-2852, 2016.

15. Pan XP and Ni ZZ: Application of intraclass correlation coefficient to reliability assessment. Hua Xi Yi Ke Da Xue Xue Bao 30: 62-63, 1999 (In Chinese).

16. Szczepanek-Parulska E, Woliński K, Stangierski A, Gurgul E and Ruchała M: Biochemical and ultrasonographic parameters influencing thyroid nodules elasticity. Endocrine 47: 519-527, 2014.

17. Bhatia KS, Rasalkar DP, Lee YP, Wong KT, King AD, Yuen HY and Ahuja AT: Cystic change in thyroid nodules: A confounding factor for real-time qualitative thyroid ultrasound elastography. Clin Radiol 66: 799-807, 2011.
18. Cengic I, Tureli D, Altas H, Ozden F, Bugdayci O and Aribal E: Effects of nodule characteristics on sampling number and duration of thyroid fine-needle aspiration biopsy: Size does not matter, but cystic degeneration ratio does. Acta Radiol 58: 286-291, 2016.

19. Gu WJ, Zhao L, Zhu XX, Tang Z, Luo Y, Wang F, Yang G, Jin $\mathrm{Z}$ and Du J: Retrospective analysis of sonographic features in 2453 thyroid nodules. Chin J Endocrinol Metab 29: 548-552, 2013.

20. Chan BK, Desser TS, Mc Dougall IR, Weigel RJ and Jeffrey RB Jr: Common and uncommon sonographic features of papillary thyroid carcinoma. J Ultrasound Med 22: 1083-1090, 2013.

21. Wharry LI, McCoy KL, Stang MT, Armstrong MJ, LeBeau SO, Tublin ME, Sholosh B, Silbermann A, Ohori NP, Nikiforov YE, et al: Thyroid nodules $(\geq 4 \mathrm{~cm})$ : Can ultrasound and cytology reliably exclude cancer? World J Surg 38: 614-621, 2014.

22. Yamanaka N, Kaminuma C, Taketomi-Takahashi A and Tsushima Y: Reliable measurement by virtual touch tissue quantification with acoustic radiation force impulse imaging phantom study. J Ultrasound Med 31: 1239-1244, 2012.

23. Potthoff A, Attia D, Pischke S, Kirschner J, Mederacke I, Wedemeyer H, Manns MP, Gebel MJ and Rifai K: Influence of different frequencies and insertion depths on the diagnostic accuracy of liver elastography by acoustic radiation force impulse imaging (ARFI). Eur J Radiol 82: 1207-1212, 2013.

24. Wang CZ, Zheng J, Huang ZP, Xiao Y, Song D, Zeng J, Zheng HR and Zheng RQ: Influence of measurement depth on the stiffness assessment of healthy liver with real-time shear wave elastography. Ultrasound Med Biol 40: 461-469, 2014.

25. Yoon JH, Jung HK, Lee JT and Ko KH: Shear-wave elastography in the diagnosis of solid breast masses: What leads to false-negative or false-positive results? Eur Radiol 23: 2432-2440, 2013.

26. Barr RG, Memo R and Schaub CR: Shear wave ultrasound elastography of the prostate: Initial results. Ultrasound Q 28: 13-20, 2012.

27. Bhatia KSS, Lee YYP, Yuen EHY and Ahuja AT: Ultrasound elastography in the head and neck. Part II. Accuracy for malignancy. Cancer Imaging 13: 260-276, 2013.

28. Liu B, Liang J, Zheng Y, Xie X, Huang G, Zhou L, Wang W and $\mathrm{Lu} \mathrm{M}$ : Two-dimensional shear wave elastography as promising diagnostic tool for predicting malignant thyroid nodules: A prospective single-centre experience. Eur Radiol 25: 624-634, 2015.

29. Duan SB, Yu J, Li X, Han ZY, Zhai HY and Liang P: Diagnostic value of two-dimensional shear wave elastography in papillary thyroid microcarcinoma. Onco Targets Ther 9: 1311-1317, 2016

30. Ruchala M, Szczepanek-Parulska E, Zybek A, Moczko J, Czarnywojtek A, Kaminski G and Sowinski J: The role of sonoelastography in acute, subacute and chronic thyroiditis: A novel application of the method. Eur J Endocrinol 166: 425-432, 2012.

31. Magri F, Chytiris S, Capelli V, Alessi S, Nalon E, Rotondi M, Cassibba S, Calliada F and Chiovato L: Shear wave elastography in the diagnosis of thyroid nodules: Feasibility in the case of coexistent chronic autoimmune Hashimoto's thyroiditis. Clin Endocrinol (Oxf) 76: 137-141, 2012.

32. Hong YR, Wu YL, Luo ZY, Wu NB and Liu XM: Impact of nodular size on the predictive values of gray-scale, color-Doppler ultrasound, and sonoelastography for assessment of thyroid nodules. J Zhejiang Univ Sci B 13: 707-716, 2012.

This work is licensed under a Creative Commons Attribution-NonCommercial-NoDerivatives 4.0 International (CC BY-NC-ND 4.0) License. 